\title{
VARIATIONAL CONTROL PROBLEMS FOR LINEAR DIFFERENTIAL SYSTEMS WTTH STIELTJES BOUNDARY CONDITIONS
}

\author{
W. L. CHAN and S. K. NG
}

(Received 5 April 1978)

(Revised 13 June 1978)

\begin{abstract}
Necessary and sufficient conditions for optimality in the control of linear differential systems $\dot{x}=A x+B u$ with Stieltjes boundary conditions $\int_{t_{0}}^{t_{2}} d \nu(t) x(t)=0$, where $\nu$ is an $r \times n$ matrix valued measure of bounded variation, are obtained. Feedback-like control is given in the case of quadratic performance.
\end{abstract}

\section{Introduction}

In the last decade, the development of the relatively new field of general boundary value problems consisting of the study of an ordinary differential system under general boundary conditions and differential-boundary operators has been vigorously pursued by many, noticeably by A. Krall and his associates. Krall [5] gave a comprehensive and up-to-date survey of the state-of-art of the field. Examples of this type of system arise from various disciplines, for example, diffusion processes, dissipative operators, nuclear reactors, vibrating wires in magnetic fields to name just a few. On the other hand, results in the control of special cases of this type of system (specifically, with multipoint constraints in the state trajectory) have been reported by Bryson and Ho [4]. The purpose of this paper is to give a more precise investigation for the necessary and sufficient conditions of the optimal control of linear systems with Stieltjes boundary conditions, as studied by Brown and Krall [3], when the control is unconstrained. In particular, we give a generalized feedback structure for the quadratic performance case. An example is also given to illustrate our analytical results. 
We shall study optimal control problems of the following type. Let $\mathscr{U}_{\text {ad }}$ be the set consisting of all $u \in L_{m}^{1}\left[t_{0}, t_{1}\right]$ such that for each $u \in \mathscr{U}_{\mathrm{ad}}$ there exists $\phi$, an absolutely continuous function on $\left[t_{0}, t_{1}\right]$ with range in $R^{n}$ such that

(i)

$$
\dot{\phi}(t)=A(t) \phi(t)+B(t) u(t),
$$

where $A(t), B(t)$ are $n \times n$ and $n \times m$ continuous matrices respectively,

$$
\begin{gathered}
\phi\left(t_{0}\right)=x_{0} \quad \text { given, } \\
\int_{t_{0}}^{t_{1}} d v(t) \phi(t)=0,
\end{gathered}
$$

where $\nu$ is an $r \times n$ matrix-valued measure of bounded variation.

The pair $(\phi, u)$ is said to be an admissible pair. Let $J$ be a real-valued function on the set of all admissible pairs:

$$
J(\phi, u)=g\left(t_{1}, \phi\left(t_{1}\right)\right)+\int_{t_{0}}^{t_{1}} L(t, \phi(t), u(t)) d t,
$$

where $g(t, \cdot)$ and $L(t, \cdot)$ are $C^{2}$ on $R^{n}$ and on $R^{n} \times R^{m}$ respectively for each $t \in\left[t_{0}, t_{1}\right]$. The problem is to find $(\bar{\phi}, \bar{u})$ such that

$$
J(\bar{\phi}, \bar{u})=\min _{u \in \mathscr{\Psi}_{\text {tad }}} J(\phi, u) .
$$

In what follows, we want to characterize such an optimal pair $(\bar{\phi}, \bar{u})$.

\section{Preliminaries}

In this section we shall derive an integration by parts formula. Our development parallels that of Brown and Krall [3]. By the Lebesgue decomposition, $\nu$ can be uniquely decomposed in the form $\nu=\nu_{c}+\nu_{s}$ where $\nu_{c}$ and $\nu_{s}$ are measures of bounded variations such that $\nu_{s} \perp d t$ and $\nu_{c} \ll d t$ with $d t$ denoting the Lebesgue measure. Thus (3) can be written as

$$
\int_{t_{0}}^{t_{1}} d v_{s}(t) x(t)+\int_{b_{0}}^{t_{1}} \frac{d v_{c}}{d t} x(t) d t=0,
$$

where $d v_{c} / d t$ is the Radon-Nikodym derivative of $v_{c}$ with respect to $d t$.

Let

$$
\begin{gathered}
E=\left\{x \in L_{n}^{2}\left[t_{0}, t_{1}\right]: x \text { is absolutely continuous on }\left[t_{0}, t_{1}\right], \text { and (3) is satisfied }\right\}, \\
p=\left\{p \in L_{n}^{2}\left[t_{0}, t_{1}\right]: p(t)=h(t)-\nu_{s}^{\mathrm{T}}\left[t_{0}, t\right] b \text { for some } b \in R^{r}, h\right. \text { is } \\
\text { absolutely continuous on } \left.\left[t_{0}, t_{1}\right] \text { and } \dot{p} \in L_{n}^{1}\left[t_{0}, t_{1}\right]\right\} .
\end{gathered}
$$

where $\mathrm{T}$ denotes transpose. 
We have then

LEMMA 2.1. For $\phi \in E$ and $p \in P$, the following relation holds

$\int_{t_{0}}^{t_{1}} p^{\mathrm{T}}(t) \dot{\phi}(t) d t=p\left(t_{1}\right)^{\mathrm{T}} \phi\left(t_{1}\right)-p\left(t_{0}\right)^{\mathrm{T}} \phi\left(t_{0}\right)-b^{\mathrm{T}} \int_{t_{0}}^{t} \frac{d \nu_{c}(t)}{d t} \phi(t) d t-\int_{b_{0}}^{t_{1}} \dot{p}^{\mathrm{T}}(t) \phi(t) d t$,

where $\mathrm{T}$ denotes transpose.

Proof. We start with the equation

$$
\begin{aligned}
& \int_{t_{0}}^{t_{1}} p^{\mathrm{T}}(t) \phi(t) d t+\int_{b_{0}}^{t_{1}} \dot{p}^{\mathrm{T}}(t) \phi(t) d t \\
& =\int_{\iota_{0}}^{t_{1}}\left[p(t)+\nu_{s}^{\mathrm{T}}\left[t_{0}, t\right] b\right]^{\mathrm{T}} \phi(t) d t-\int_{\iota_{0}}^{t_{1}}\left[\nu_{s}^{\mathrm{T}}\left[t_{0}, t\right]\right]^{\mathrm{T}} \phi(t) d t \\
& \quad+\int_{t_{0}}^{t_{1}}\left[p(t)+\nu_{s}^{\mathrm{T}}\left[t_{0}, t\right] b\right]^{\mathrm{T}^{\prime}} \phi(t) d t-\int_{t_{0}}^{t_{1}}\left[\nu_{s}^{\mathrm{T}}\left[t_{0}, t\right] b\right]^{\mathrm{T}^{\prime}} \phi(t) d t,
\end{aligned}
$$

where ()$^{\prime}$ also means differentiation.

This is possible since $\nu_{s}^{\mathrm{T}}\left[t_{0}, t\right] b$ is differentiable a.e. on $\left[t_{0}, t_{1}\right]$ and $\left(\nu_{s}^{\mathrm{T}}\left[t_{0}, t_{1}\right] b\right)^{\prime} \in L_{n}^{1}\left[t_{0}, t_{1}\right]$. The right-hand side of $(7)$ now equals

$$
\begin{aligned}
{\left[p\left(t_{1}\right)\right.} & \left.+\nu_{s}^{\mathrm{T}}\left[t_{0}, t_{1}\right] b\right]^{\mathrm{T}} \phi\left(t_{1}\right)-\left[p\left(t_{0}\right)+\nu_{s}^{\mathrm{T}}\left[t_{0}\right] b\right]^{\mathrm{T}} \phi\left(t_{0}\right) \\
& -\int_{b_{0}}^{t_{1}}\left[p(t)+\nu_{s}^{\mathrm{T}}\left[t_{0}, t\right] b\right]^{\mathrm{T}^{\prime}} \phi(t) d t-\int_{b_{0}}^{t_{1}}\left[\nu_{s}^{\mathrm{T}}\left[t_{0}, t\right] b\right]^{\mathrm{T}} \phi(t) d t \\
& +\int_{b_{0}}^{t_{1}}\left[p(t)+\nu_{s}^{\mathrm{T}}\left[t_{0}, t\right] b\right]^{\mathrm{T}^{\prime}} \phi(t) d t+0
\end{aligned}
$$

Since $p(t)+\nu_{s}^{\mathrm{T}}\left[t_{0}, t\right] b$ is absolutely continuous on $\left[t_{0}, t_{1}\right]$, the first three terms are obtained by integration by parts on the first term of the right-hand side of (7); and since $\nu_{s} \perp d t$ and we have that $\nu_{s} \perp d t$ if and only if $\left(d v_{s} / d t\right)\left[t_{0}, t\right]=0$, a.e. the fourth term of the right-hand side of (7) is zero.)

Therefore the right-hand side of $(7)$ becomes

$$
\begin{aligned}
& {\left[p\left(t_{1}\right)+\nu_{s}^{\mathrm{T}}\left[t_{0}, t_{1}\right] b\right]^{\mathrm{T}} \phi\left(t_{1}\right)-\left[p\left(t_{0}\right)+\nu_{s}^{\mathrm{T}}\left[t_{0}\right] b\right]^{\mathrm{T}} \phi\left(t_{0}\right)} \\
& -b^{\mathrm{T}}\left[\nu_{s}^{\mathrm{T}}\left[t_{0}, t_{1}\right]^{\mathrm{T}} \phi\left(t_{1}\right)-\nu_{s}^{\mathrm{T}}\left[t_{0}\right]^{\mathrm{T}} \phi\left(t_{0}\right)-\int_{b_{0}}^{t_{1}} d \nu_{s}(t) \phi(t)\right] \\
& =p\left(t_{1}\right)^{\mathrm{T}} \phi\left(t_{1}\right)-p\left(t_{0}\right)^{\mathrm{T}} \phi\left(t_{0}\right)+b^{\mathrm{T}} \int_{t_{0}}^{t_{1}} d \nu_{s}(t) \phi(t) \\
& =p\left(t_{1}\right)^{\mathrm{T}} \phi\left(t_{1}\right)-p\left(t_{0}\right)^{\mathrm{T}} \phi\left(t_{0}\right)-b^{\mathrm{T}} \int_{b_{0}}^{t_{1}} \frac{d \nu_{c}(t)}{d t} \phi(t) d t .
\end{aligned}
$$




\section{Necessary conditions for optimality}

The following development is an extension of those in [4, pp. 47-59] and [2, sections 5-7]. Suppose that $(\bar{p}, \bar{u})$ is a local solution to (5). Let $u=\bar{u}+\varepsilon \delta u \in \mathscr{U}_{\text {ad }}$ and $\phi$ corresponds to $\bar{u}+\varepsilon \delta u$. Then we know

$$
\bar{\phi}(t)=\Phi\left(t, t_{0}\right) x_{0}+\int_{\omega_{0}}^{t} \Phi(t, \tau) B(\tau) \bar{u}(\tau) d \tau
$$

and

$$
\phi(t)=\Phi\left(t, t_{0}\right) x_{0}+\int_{b_{0}}^{t} \Phi(t, \tau) B(\tau)(\tilde{u}(\tau)+\varepsilon \delta u(\tau)) d \tau,
$$

where $\Phi(t, \tau)$ is the fundamental matrix of $\dot{x}=A x$. Let

$$
\varepsilon \delta \phi(t) \equiv \phi(t)-\phi(t)=\varepsilon \int_{\iota_{0}}^{t} \Phi(t, \tau) B(\tau) \delta u(\tau) d \tau .
$$

Noting that $\delta \phi\left(t_{0}\right)=0$ and

$$
\varepsilon \int_{t_{0}}^{t_{1}} d \nu(t) \delta \phi(t)=\int_{t_{0}}^{t_{1}} d \nu(t) \phi(t)-\int_{t_{0}}^{t_{1}} d \nu(t) \Phi(t)=0,
$$

we find

$$
\int_{t_{0}}^{t_{1}} d \nu(t) \delta \phi(t)=0 .
$$

Now consider the value $J(\bar{\phi}+\varepsilon \delta \phi, \bar{u}+\varepsilon \delta u)-J(\bar{\phi}, \bar{u})$. Let

$$
H(t, x, u, p)=L(t, x, u)+p^{\mathrm{T}} f(t, x, u)
$$

with

$$
f(t, x, u) \equiv A x+B u, \quad p \in R^{n} .
$$

Then, along the path $(\bar{\phi}(t), \bar{u}(t)), t \in\left[t_{0}, t_{1}\right]$ and $p \in P$,

$$
\begin{aligned}
& J(\bar{\phi}+\varepsilon \delta \phi, \bar{u}+\varepsilon \delta u)-J(\bar{\phi}, \bar{u}) \\
& =g\left(t_{1}, \phi\left(t_{1}\right)\right)-g\left(t_{1}, \Phi\left(t_{1}\right)\right)+\int_{t_{0}}^{t_{1}}[L(t, \phi(t), u(t))-L(t, \bar{\phi}(t), \bar{u}(t))] d t \\
& =\varepsilon\left(\left.\frac{\partial g}{\partial x}\right|_{\Lambda}\right)^{\mathrm{T}} \delta \phi\left(t_{1}\right)+o(\varepsilon)+\int_{t_{0}}^{t_{1}}[H(t, \bar{\phi}+\varepsilon \delta \phi, \bar{u}+\varepsilon \delta u, p)-H(\varepsilon, \bar{\phi}, \bar{u}, \bar{p})] d t \\
& \quad+\int_{b_{0}}^{t_{1}} p^{\mathrm{T}}[-(\dot{\phi}+\varepsilon \delta \phi)+\dot{\phi}] d t,
\end{aligned}
$$

where $\left.(\partial g / \partial x)\right|_{\Lambda}$ denotes $\partial g / \partial x$ evaluated at $\left(t_{1}, \phi\left(t_{1}\right)\right)$ and $\bar{\phi}, \bar{u}, \bar{p}, H(t, \bar{\phi}, \bar{u}, \bar{p})$ are simplified notations for $\bar{\phi}(t), \bar{u}(t), \bar{p}(t), H(t, \bar{\phi}(t), \bar{u}(t), \bar{p}(t))$. The use of Lemma 2.1 
and the fact $\delta \phi\left(t_{0}\right)=0$ then gives

$$
\begin{aligned}
& J(\bar{\phi}+\varepsilon \delta \phi, \bar{u}+\varepsilon \delta u)-J(\bar{\phi}, \bar{u}) \\
& =\varepsilon\left(\left.\frac{\partial g}{\partial x}\right|_{\Lambda}\right)^{\mathrm{T}} \delta \phi\left(t_{1}\right)+\int_{t_{0}}^{t_{1}} \varepsilon\left[\left(\left.\frac{\partial H}{\partial u}\right|_{\Lambda}\right)^{\mathrm{T}} \delta u+\left(\left.\frac{\partial H}{\partial x}\right|_{\Lambda}\right)^{\mathrm{T}} \delta \phi\right] d t \\
& \quad-\varepsilon\left\{p\left(t_{1}\right)^{\mathrm{T}} \delta \phi\left(t_{1}\right)-b^{\mathrm{T}} \int_{l_{0}}^{t_{1}} \frac{d \nu_{c}}{d t} \delta \phi d t-\int_{l_{0}}^{t_{1}} \dot{p}^{\mathrm{T}} \delta \phi d t\right\}+o(\varepsilon) \\
& =\varepsilon\left(\left(\left.\frac{\partial g}{\partial x}\right|_{\Lambda}\right)^{\mathrm{T}}-p\left(t_{1}\right)^{\mathrm{T}}\right) \delta \phi\left(t_{1}\right)+\int_{l_{0}}^{t_{1}} \varepsilon\left[\left(\left.\frac{\partial H}{\partial x}\right|_{\Lambda}\right)^{\mathrm{T}}+b^{\mathrm{T}} \frac{d \nu_{c}}{d t}+\dot{p}^{\mathrm{T}}\right] \delta \phi d t \\
& +\varepsilon \int_{t_{0}}^{t_{1}}\left(\left.\frac{\partial H}{\partial u}\right|_{\Lambda}\right)^{\mathrm{T}} \delta u d t+o(\varepsilon),
\end{aligned}
$$

On the other hand, let $p$ be such that

$$
\begin{aligned}
& -\dot{p}=\left.\frac{\partial H}{\partial x}\right|_{\Lambda}+\frac{d \nu_{c}^{\mathrm{T}}}{d t} b \quad \text { a.e. on }\left[t_{0}, t_{1}\right] \\
& p\left(t_{1}\right)=\left.\frac{\partial g}{\partial x}\right|_{\Lambda}
\end{aligned}
$$

then, from (8) and using the fact that $J(\bar{\phi}, \bar{u})$ is a local minimum, we have

$$
\int_{b_{0}}^{t_{1}}\left(\left(\left.\frac{\partial L}{\partial u}\right|_{\Lambda}\right)^{\mathrm{T}}+p^{\mathrm{T}} B\right) \delta u d t=\int_{t_{0}}^{t_{1}}\left(\left.\frac{\partial H}{\partial u}\right|_{\Lambda}\right)^{\mathrm{T}} \delta u d t=0 .
$$

Now choose $\left.\delta \bar{u} \equiv(\partial L / \partial u)\right|_{\Lambda}+B^{\mathrm{T}} p$, where $p$ is still undetermined because $b$ is arbitrary. We want to choose $p$ such that $\delta \tilde{u}$ is admissible, that is,

$$
\int_{l_{0}}^{t_{1}} d \nu(t) \delta \bar{\phi}(t)=0
$$

where $\delta \bar{\phi}$ corresponds to $\delta \bar{u}$. Further, (12) is easily seen to be equivalent to

$$
\int_{l_{0}}^{t_{1}} d \nu(t) \int_{l_{0}}^{t^{\prime}} \Phi(t, \tau) B B^{\mathrm{T}} p d \tau=-\left.\int_{t_{0}}^{t_{1}} d \nu(t) \int_{l_{0}}^{t} \Phi(t, \tau) B \frac{\partial L}{\partial u}\right|_{\Lambda} d \tau
$$

Now let $\bar{p}(t)$ be defined as

$$
\begin{aligned}
\bar{p}(t) \equiv \Phi^{\mathrm{T}}\left(t_{1}, t\right) & {\left[\left.\frac{\partial g}{\partial x}\right|_{\Lambda}+\nu_{s}^{\mathrm{T}}\left[t_{0}, t_{1}\right] b\right]-\nu_{s}^{\mathrm{T}}\left[t_{0}, t\right] b } \\
& +\int_{t_{1}}^{t} \Phi^{\mathrm{T}}(\tau, t)\left[-\left.\frac{\partial L}{\partial x}\right|_{\Lambda}-\frac{d \nu_{c}^{\mathrm{T}}}{d t} b+A^{\mathrm{T}} \nu_{s}^{\mathrm{T}}\left[t_{0}, \tau\right] b\right] d \tau
\end{aligned}
$$


on $\left[t_{0}, t_{1}\right]$. Then, $\bar{p}$ is of the form $\bar{p}(t)=h(t)-\nu_{s}^{\mathrm{T}}\left[t_{0}, t\right] b$ and hence $\bar{p} \in P$. Further, note that

$$
\begin{aligned}
\bar{p}\left(t_{1}\right) & =\Phi^{\mathrm{T}}\left(t_{1}, t_{1}\right)\left[\left.\frac{\partial g}{\partial x}\right|_{\Lambda}+\nu_{s}^{\mathbf{T}}\left[t_{0}, t_{1}\right] b\right]-\nu_{s}^{\mathrm{T}}\left[t_{0}, t_{1}\right] b \\
& =\left.\frac{\partial g}{\partial x}\right|_{\Lambda} .
\end{aligned}
$$

Also, since $d / d t\left(\nu_{s}^{\mathbf{T}}\left[t_{0}, t\right] b\right)$ exists and is equal to zero a.e. on $\left[t_{0}, t_{1}\right]$ it follows that $\hat{p}(t)$ exists for almost all $t$ in $\left[t_{0}, t_{1}\right]$ and

$$
\begin{aligned}
\dot{\bar{p}}(t)= & -A^{\mathrm{T}} \Phi\left(t_{1}, t\right)\left[\left.\frac{\partial g}{\partial x}\right|_{\Lambda}+\nu_{s}^{\mathrm{T}}\left[t_{0}, t_{1}\right] b\right]+\left\{-\left.\frac{\partial L}{\partial x}\right|_{\Lambda}-\frac{d \nu_{c}^{\mathrm{T}}}{d t} b+A^{\mathrm{T}} \nu_{s}^{\mathrm{T}}\left[t_{0}, t\right] b\right\} \\
& +\int_{t_{1}}^{t}-A^{\mathrm{T}} \Phi^{\mathrm{T}}(\tau, t)\left[-\left.\frac{\partial L}{\partial x}\right|_{\Lambda}-\frac{d \nu_{c}^{\mathrm{T}}}{d t} b+A^{\mathrm{T}} \nu_{s}^{\mathrm{T}}\left[t_{0} \tau\right] b\right] d \tau \\
= & -A^{\mathrm{T}}(t) \bar{p}(t)+\left\{-\left.\frac{\partial L}{\partial x}\right|_{\mathrm{V}}-\frac{d \nu_{c}^{\mathrm{T}}}{d t} b\right\} \\
= & -\left.\frac{\partial H}{\partial x}\right|_{\Lambda}-\frac{d \nu_{c}^{\mathrm{T}}}{d t} b \quad \text { a.e. on }\left[t_{0}, t_{1}\right] .
\end{aligned}
$$

Thus $\bar{p}(t)$ satisfies the conditions (9) and (10). Finally, from (12) or (13), we require $\bar{p}$ to be such that

$$
\begin{gathered}
\int_{t_{0}}^{t_{1}} d \nu(t) \int_{l_{0}}^{t} \Phi(t, \tau) B B^{\mathrm{T}}\left[\Phi^{\mathrm{T}}\left(t_{1}, \tau\right)\left(\left.\frac{\partial g}{\partial x}\right|_{\Lambda}+\nu_{s}^{\mathrm{T}}\left[t_{0}, t_{1}\right] b\right)\right. \\
\left.+\int_{t_{1}}^{\tau} \Phi^{\mathrm{T}}(s, \tau)\left(\left.\frac{\partial L}{\partial x}\right|_{\Lambda}-\frac{d \nu_{c}^{\mathrm{T}}}{d t} b+A^{\mathrm{T}} \nu_{s}^{\mathrm{T}}\left[t_{0}, s\right] b\right) d s-\nu_{s}^{\mathrm{T}}\left[t_{0}, \tau\right] b\right] d \tau \\
=-\left.\int_{t_{0}}^{t_{1}} d \nu(t) \int_{\iota_{0}}^{t} \Phi(t, \tau) B \frac{\partial L}{\partial u}\right|_{\Lambda} d \tau .
\end{gathered}
$$

In other words, we have to solve for $b$ in the following system of linear equations:

$$
\begin{gathered}
\int_{t_{0}}^{t_{1}} d \nu(t) \int_{t_{0}}^{t} \Phi(t, \tau) B B^{\mathrm{T}}\left[\phi^{\mathrm{T}}\left(t_{1}, \tau\right) \nu_{s}^{\mathrm{T}}\left[t_{0}, t_{1}\right]\right. \\
\left.+\int_{t_{1}}^{\tau} \Phi^{\mathrm{T}}(s, \tau)\left\{\frac{d \nu_{c}^{\mathrm{T}}}{d t}+A^{\mathrm{T}} \nu_{s}^{\mathrm{T}}\left[t_{0}, s\right]\right\} d s-\nu_{s}^{\mathrm{T}}\left[t_{0}, \tau\right]\right] d \tau \cdot b \\
=-\int_{l_{0}}^{t_{1}} d \nu(t) \int_{t_{0}}^{t} \Phi(t, \tau) B B^{\mathrm{T}}\left[\left.\Phi^{\mathrm{T}}\left(t_{1}, \tau\right) \frac{\partial g}{\partial x}\right|_{\Lambda}-\left.\int_{t_{1}}^{\tau} \Phi^{\mathrm{T}}(s, \tau) \frac{\partial L}{\partial x}\right|_{\Lambda} d s\right] d \tau \\
-\left.\int_{l_{0}}^{t_{1}} d \nu(t) \int_{l_{0}}^{t} \Phi(t, \tau) B \frac{\partial L}{\partial u}\right|_{\Lambda} d \tau .
\end{gathered}
$$


The above equation can be written as

$$
M b=k,
$$

where $M$ is an $r \times r$ matrix and $k$ is an $r$-vector.

Assumption 3.1. Either $\nu \equiv 0$ or $M$ is nonsingular.

If Assumption 3.1 holds, then (16) has a solution $b$. It follows that $\bar{p}$ is thus determined. With this $\bar{p}$, we have that

$$
\int_{l_{0}}^{l_{1}} d \nu(t) \delta \phi(t)=0,
$$

and hence $\delta \bar{u}=\left.(\partial L / \partial u)\right|_{\Lambda}+B^{\mathrm{T}} \bar{p}$ is admissible and, from (11),

$$
\begin{aligned}
0 & =\int_{\iota_{0}}^{t_{2}}\left(\left(\left.\frac{\partial L}{\partial u}\right|_{\Lambda}\right)^{\mathrm{T}}+\bar{p}^{\mathrm{T}} B\right) \delta \bar{u} d t=\int_{\iota_{0}}^{t_{1}}\left(\left(\left.\frac{\partial L}{\partial u}\right|_{\Lambda}\right)^{\mathrm{T}}+\bar{p}^{\mathrm{T}} B\right)\left(\left.\frac{\partial L}{\partial u}\right|_{\Lambda}+B^{\mathrm{T}} \bar{p}\right) d t \\
& =\int_{\iota_{0}}^{t_{1}}\left\|\left.\frac{\partial L}{\partial u}\right|_{\Lambda}+B^{\mathrm{T}} \bar{p}\right\|^{2} d t
\end{aligned}
$$

This shows that

$$
0=\left.\frac{\partial H}{\partial u}\right|_{\Lambda}=\left.\frac{\partial L}{\partial u}\right|_{\Lambda}+B^{\mathrm{T}} \bar{p} \quad \text { a.e. on }\left[t_{0}, t_{1}\right]
$$

Summarizing, we have proved the following

THEOREM 3.1. Suppose Assumption 3.1 holds, then for $(\bar{\phi}, \bar{u})$ to be a solution of (5), it is necessary that there exists a $\bar{p} \in P$ such that the following relations hold:

$$
\begin{gathered}
\dot{\phi}=\left.\frac{\partial H}{\partial p}\right|_{\Lambda}=A \Phi+B \bar{u}, \quad \Phi\left(t_{0}\right)=x_{0}, \\
\int_{t_{0}}^{t_{1}} d \nu(t) \Phi(t)=0 ; \\
\dot{\bar{p}}=-\left.\frac{\partial H}{\partial x}\right|_{\Lambda}-\frac{d \nu_{c}^{\mathrm{T}}}{d t} b=-A^{\mathrm{T}} \bar{p}-\left.\frac{\partial L}{\partial x}\right|_{\Lambda}-\frac{d \nu_{c}^{\mathrm{T}}}{d t} b,
\end{gathered}
$$

a.e. on $\left[t_{0}, t_{1}\right]$ where $b$ is a constant vector to be determined and

$$
\bar{p}\left(t_{1}\right)=\left.\frac{\partial g}{\partial x}\right|_{\Lambda}
$$


Indeed, $\bar{p}$ is of the form:

$$
\begin{aligned}
\bar{p}(t)=\Phi^{\mathrm{T}}\left(t_{1}, t\right)\left\{\left.\frac{\partial g}{\partial x}\right|_{\Lambda}+\nu_{s}^{\mathrm{T}}\left[t_{0}, t_{1}\right] b\right\}-\nu_{s}^{\mathrm{T}}\left[t_{0}, t\right] b \\
\\
+\int_{t_{1}}^{t} \Phi^{\mathrm{T}}(\tau, t)\left\{-\left.\frac{\partial L}{\partial x}\right|_{\Lambda}-\frac{d \nu_{c}^{\mathrm{T}}}{d t} b+A^{\mathrm{T}} \nu_{s}^{\mathrm{T}}\left[t_{0}, \tau\right] b\right\} d \tau,
\end{aligned}
$$

where $\Phi$ is the fundamental matrix of (1). Further,

$$
\left.\frac{\partial H}{\partial u}\right|_{\Lambda}=\left.\frac{\partial L}{\partial u}\right|_{\Lambda}+B^{\mathrm{T}} \bar{p}=0 \text { a.e. on }\left[t_{0}, t_{1}\right]
$$

where $H(t, x, u, p) \equiv L(t, x, u)+p^{\mathrm{T}}(A x+B u)$.

We now give conditions under which Theorem 3.1 becomes sufficient.

COROLlaRY 3.1. Suppose Assumption 3.1 holds and that $J$ is convex on the set of all admissible pairs. Then, in order for $(\bar{\phi}, \bar{u})$ to be a solution of $(5)$, it is necessary and sufficient that the relations in Theorem 3.1 hold.

ProOF. By Theorem 3.1, we need only to prove the sufficient part.

Suppose the relations in Theorem 3.1 hold. Then this means that

$$
J(\Phi+\varepsilon(\phi-\bar{\phi}), \bar{u}+\varepsilon(u-\bar{u}))-J(\bar{\phi}, \bar{u}) \geqslant o(\varepsilon) \text { for all } u \in \mathscr{U}_{\mathrm{ad}} .
$$

Since $J$ is convex with respect to $(\phi, u)$, we have

$$
J(\phi, u)-J(\bar{\phi}, \bar{u}) \geqslant 1 / \varepsilon[J(\bar{\phi}+\varepsilon(\phi-\bar{\phi}), \bar{u}+\varepsilon(u-\bar{u})-J(\bar{\phi}, \bar{u})] \text { for all } 0<\varepsilon<1 \text {. }
$$

Hence $J(\phi, u)-J(\bar{\phi}, \bar{u}) \geqslant o(\varepsilon) / \varepsilon$. Letting $\varepsilon \rightarrow 0$ we have $J(\phi, u) \geqslant J(\bar{\phi}, \bar{u})$.

RemarK. Assumption 3.1 may be regarded as an extended controllability condition by the following observation.

First, from the derivation of Theorem 3.1 we see that if the constraint $\int_{t_{0}}^{t_{1}} d v(t) \phi(t)=0$ is replaced by $\int_{t_{0}}^{t_{1}} d \nu(t) \phi(t)=c$, where $c$ is a constant vector, then the above procedure remains true.

Now, in the particular case when $v_{c}=0$, we have $v_{s}\left\{t_{1}\right\}=D$ which is an $r \times n$ matrix of the form

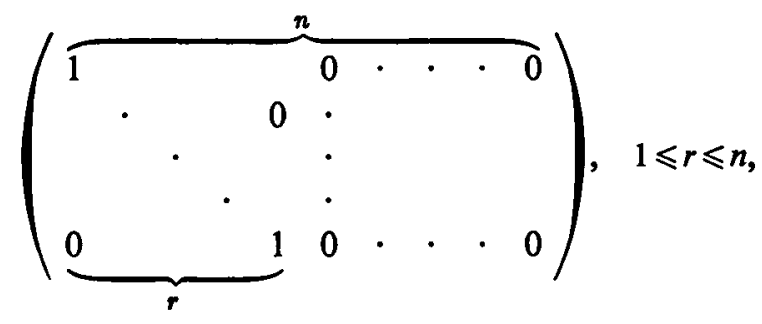


Since $\nu_{s}\{S\}=0$ whenever $S$ does not contain $t_{1}$, we may choose the constant $c$ suitably, and our problem becomes a problem with the $i$ th components $(i=1, \ldots, r)$ of the terminal state fixed.

Finally, $M$ becomes

$$
D \int_{l_{0}}^{t_{1}} \Phi\left(t_{1}, \tau\right) B(\tau) B^{\mathrm{T}}(\tau) \Phi^{\mathrm{T}}\left(t_{1}, \tau\right) d \tau D^{\mathrm{T}},
$$

which is nonsingular if $\int_{t_{0}}^{t_{1}} \Phi\left(t_{1}, \tau\right) B(\tau) B^{\mathrm{T}}(\tau) \phi^{\mathrm{T}}\left(t_{1}, \tau\right) d \tau$ is nonsingular, and the latter is the usual controllability condition.

\section{Linear quadratic problem}

For the important special case when $J$ is quadratic, we have

Proposition 4.1. Suppose Assumption 3.1 holds, and that $J$ is given by

$$
J(\phi, u)=\frac{1}{2}\left\langle\phi\left(t_{1}\right), \quad F \phi\left(t_{1}\right)\right\rangle+\frac{1}{2} \int_{\iota_{0}}^{t_{1}}\{\langle\phi(t), Q \phi(t)\rangle+\langle u, R(t) u\rangle\} d t,
$$

where $\langle\cdot, \cdot\rangle$ denotes the inner product of $R^{n}$ or $R^{m}$ and $F, Q(t) \geqslant 0, R(t)>0$ for amost all $t$ in $\left[t_{0}, t_{1}\right]$. Then, in order for $(\Phi, u)$ to be a solution of $(5)$, it is necessary and sufficient that $(\bar{\phi}, \bar{u})$ satisfies the following differential-boundary systems:

$$
\begin{gathered}
\dot{\phi}=A \phi+B u, \quad \phi\left(t_{0}\right)=x_{0}, \\
\int_{b_{0}}^{t_{1}} d \nu(t) \phi(t)=0, \\
\dot{p}=-A^{\mathrm{T}} p-Q \phi-\frac{d \nu_{c}^{\mathrm{T}}}{d t} b, \\
p\left(t_{1}\right)=F \phi\left(t_{1}\right), \\
u=-R^{-1} B^{\mathrm{T}} p .
\end{gathered}
$$

Proof. The above proposition follows from Corollary 3.1 and the fact that $J$ is convex on the set of all admissible pairs.

Next, we want to construct a feedback structure for the optimal control. We start by assuming that

$$
p(t)=K(t) \phi(t)+f(t)-\nu_{s}^{\mathrm{T}}\left[t_{0}, t\right] b,
$$

where $K(t), f(t)$ are to be determined. Differentiating (23) (thanks to the fact that $\left(d \nu_{s}^{\mathrm{T}} / d t\right)\left[t_{0}, t\right] b=0$, a.e. on $\left.\left[t_{0}, t_{1}\right]\right)$ we get

$$
\dot{p}=K \dot{\phi}+\dot{K} \phi+f \text { a.e. on }\left[t_{0}, t_{1}\right] \text {. }
$$


Employing (20-22), equation (24) becomes

$$
-A^{\mathrm{T}} p-Q \phi-\frac{d \nu_{c}^{\mathrm{T}}}{d t} b=K\left(A \phi-B R^{-1} B^{\mathrm{T}} p\right)+\dot{K} \phi+f, \quad \text { a.e. on }\left[t_{0}, t_{1}\right]
$$

or

$$
\begin{aligned}
& \left(\dot{K}+K A+A^{\mathrm{T}} K+Q-K B R^{-1} B^{\mathrm{T}} K\right) \phi+\left(A^{\mathrm{T}}-K B R^{-1} B^{\mathrm{T}}\right) f \\
& \quad-\left(A^{\mathrm{T}}-K B R^{-1} B^{\mathrm{T}}\right) \nu_{s}^{\mathrm{T}}\left[t_{0}, t\right] b+\frac{d \nu_{c}^{\mathrm{T}}}{d t} b+f=0, \text { a.e. on }\left[t_{0}, t_{1}\right]
\end{aligned}
$$

Now choose $K(\cdot)$ to be an absolutely continuous matrix-valued function such that

$$
\begin{aligned}
\dot{K} & =-K A-A^{\mathrm{T}} K+K B R^{-1} B^{\mathrm{T}} K-Q, \\
K\left(t_{1}\right) & =F
\end{aligned}
$$

and $f(\cdot)$ to be an absolutely continuous function from $\left[t_{0}, t_{1}\right]$ to $R^{n}$ such that

$$
\begin{aligned}
f & =\left(K B R^{-1} B^{\mathrm{T}}-A^{\mathrm{T}}\right) f-\left[\left(K B R^{-1} B^{\mathrm{T}}-A^{\mathrm{T}}\right) \nu_{s}^{\mathrm{T}}\left[t_{0}, t\right]+\frac{d \nu_{c}^{\mathrm{T}}}{d t}\right] b, \\
f\left(t_{1}\right) & =\nu_{s}^{\mathrm{T}}\left[t_{0}, t_{1}\right] b .
\end{aligned}
$$

Having chosen $K(\cdot)$ and $f(\cdot)$, an optimal feedback-like control is then given by

$$
u(\phi(t), t)=-R(t)^{-1} B^{\mathrm{T}}(t)\left(K(t) \phi(t)+f(t)-\nu_{s}^{\mathrm{T}}\left[t_{0}, t\right] b\right) .
$$

Remark. For the development of $K$, one may consult (2, sections 9-3) and [1, pp. 23-26].

We note that the existence of solution of the Riccati equation (26) is independent of $\nu$, and one may show that this existence is implied by the existence of minimal $J$ with $\nu=0$, which is always the case (see, for example, [1, pp. 2j-28]).

To illustrate the results of this paper we give a simple example.

Minimize

$$
J(\phi, u)=\frac{1}{2} \phi(2)^{2}+\frac{1}{2} \int_{0}^{2} u^{2}(t) d t
$$

subject to

$$
\begin{gathered}
\phi=u, \quad \phi(0)=\alpha, \\
\phi(0)+\phi(1)+\phi(2)=0 .
\end{gathered}
$$

First note that $M=5$ so that Assumption 3.1 holds. Let

and we have

$$
H=\frac{1}{2} u^{2}+p u
$$

$$
\bar{p}(t)=\left(\bar{\phi}(2)+\nu_{s}^{\mathrm{T}}[0,2] b\right)-\nu_{s}^{\mathrm{T}}[0, t] b,
$$


where $\nu_{s}$ is a measure such that $\nu_{s}\{0\}=\nu_{s}\{1\}=\nu_{s}\{2\}=1$, and $\nu_{s}\{A\}=0$ for all measurable sets $A$ in $R$ whenever $A \cap\{0,1,2\}$ is empty. Then,

$$
\bar{p}(t)= \begin{cases}\bar{\phi}(2)+2 b, & 0 \leqslant t<1, \\ \bar{\phi}(2)+b, & 1 \leqslant t<2, \\ \bar{\phi}(2), & t=2,\end{cases}
$$

and $\partial H / \partial u=\bar{u}+\bar{p}=0$ a.e. implies

$$
\bar{u}(t)=-\bar{p}(t)= \begin{cases}-\bar{\phi}(2)-2 b, & 0 \leqslant t<1, \\ -\bar{\phi}(2)-b, & 1 \leqslant t<2, \\ -\bar{\phi}(2), & t=2 .\end{cases}
$$

After some calculations, we have

$$
\begin{aligned}
& \bar{\phi}(1)=\frac{2}{3} \bar{\phi}(0)-b, \\
& \phi(2)=\frac{1}{3} \phi(0)-b .
\end{aligned}
$$

To find $b$, let $\phi(0)+\phi(1)+\bar{\phi}(2)=0$ so that $b=\bar{\phi}(0)=\alpha$, and hence

$$
\bar{u}(t)= \begin{cases}-\frac{4}{3} \alpha, & 0 \leqslant t<1, \\ -\frac{1}{3} \alpha, & 1 \leqslant t \leqslant 2 .\end{cases}
$$

Also evaluating $J$ gives

$$
J(\bar{\phi}, \bar{u})=\frac{21}{18} \alpha^{2} .
$$

Furthermore, we see that $(\bar{\phi}, \bar{u})$ is an optimal solution by the following observation.

Let

$$
\begin{aligned}
u(t) & = \begin{cases}\bar{u}(t)+k_{1}(t), & 0 \leqslant t<1, \\
\bar{u}(t)+k_{2}(t), & 1 \leqslant t \leqslant 2,\end{cases} \\
& = \begin{cases}-\frac{4}{3} \alpha+k_{1}(t), & 0 \leqslant t<1, \\
-\frac{1}{3} \alpha+k_{2}(t), & 1 \leqslant t \leqslant 2 .\end{cases}
\end{aligned}
$$

Then, in order to satisfy $\phi(0)+\phi(1)+\phi(2)=0$, we require

$$
2 \int_{0}^{1} k_{1}(t) d t+\int_{1}^{2} k_{2}(t) d t=0
$$

This relation leads to

$$
J(\phi, u)=J(\Phi, \bar{u})+\frac{1}{2}\left(\int_{0}^{1} k_{1}(t) d t+\int_{1}^{2} k_{2}(t) d t\right)^{2}+\int_{0}^{1} k_{1}^{2}(t) d t+\int_{1}^{2} k_{2}^{2}(t) d t \geqslant J(\Phi, \bar{u}) .
$$


To determine a closed-loop optimal control, we finally have to solve equations (26) and (27). This gives

$$
\begin{aligned}
& K(t)=\frac{1}{3-t}, \quad t \in[0,2], \\
& f(t)= \begin{cases}\frac{(6-t) b}{3-t}, & 0 \leqslant t<1, \\
\frac{(7-2 t) b}{3-t}, & 1 \leqslant t \leqslant 2,\end{cases}
\end{aligned}
$$

and

$$
\begin{aligned}
\bar{u}(\phi(t), t) & =-\left\{K(t) \phi(t)+f(t)-\nu_{s}^{\mathrm{T}}\left[t_{0}, t\right] b\right\} \\
& = \begin{cases}\frac{\phi(t)}{t-3}+\frac{(6-t) b}{t-3}+b, & 0 \leqslant t<1, \\
\frac{\phi(t)}{t-3}+\frac{(7-2 t) b}{t-3}+2 b, & 1 \leqslant t \leqslant 2,\end{cases}
\end{aligned}
$$

where $b$ is to be determined by the boundary conditions.

\section{Conclusions}

We have studied the problem of optimal control for linear systems with Stieltjes boundary conditions with unconstrained controls. The necessary conditions for optimality are represented by a system of differential-boundary equations. Under some convexity requirement on $J$, these become sufficient as well. When $J$ is quadratic, an optimal feedback control has been constructed and an example has been presented to illustrate our results.

\section{References}

[1] B. D. O. Anderson and J. B. Moore, Linear optimal control (Englewood Cliffs, N.J.: Prentice-Hall, Inc., 1971).

[2] M. Athans and P. L. Falb, Optimal control (New York: McGraw-Hill, 1966).

[3] R. C. Brown and A. M. Krall, "Ordinary differential operators under stieltjes boundary conditions", Trans. Amer. Math. Soc. 198 (1974), 73-92.

[4] A. E. Bryson and Y. C. Ho, Applied optimal control (New York: Halsted Press, 1975).

[5] A. M. Krall, "The development of general differential and general differential-boundary systems", Rocky Mountain J. of Math. 5 (1975), 493-452.

\section{Department of Mathematics \\ Science Centre \\ The Chinese University of Hong Kong \\ Shatin, N.T., Hong Kong}

